\title{
Voids identification from partially overspecified boundary data
}

\author{
Amel Ben Abda ${ }^{1}$,Emna Jaiem ${ }^{2}$, Sinda Khalfallah ${ }^{3}$ and Bochra Méjri ${ }^{4}$ \\ 1,3,4 Université de Tunis El Manar, Ecole Nationale d'Ingénieurs de Tunis, LR99ES20 Modélisation Mathématique et Numérique dans \\ les Sciences de l'Ingénieur, LAMSIN, B.P. 37, 1002 Tunis, Tunisia \\ ${ }^{2}$ Université Lille 1, Batiment P2, 59655 VILLENEUVE-D'ASCQ
}

Received: 25 December 2017, Accepted: 1 April 2018

Published online: 10 December 2018.

\begin{abstract}
This work is devoted to some geometric inverse problems in linear elasticity. The problem considered is the cavities identification in mechanical structures from the knowledge of partially overdetermined boundary data, namely the displacement field and the normal component of the normal stress. We state a uniqueness result from a single pair of data under some geometrical assumptions. We propose an iterative method based on the coupling of the data completion process through the Steklov-Poincaré operator to reconstruct the shear stress and of the shape gradient method combined with the level set method to identify cavities. Numerical simulations highlight the algorithm efficiency.
\end{abstract}

Keywords: Geometric inverse problem, linear elasticity, voids identification, partially overdetermined boundary data, identifiability, data completion, shape gradient method, level set method.

\section{Introduction}

During the past few decades, special attention has been given to inverse problems in linear elasticity framework [12]. These problems can be classified as the detection of defaults (cavities, cracks or inclusions) or the reconstruction of unreachable boundary data, among others.

On one hand, the identification of flaws in mechanical structures is extremely important in the industry $[25,30]$ since it is crucial for engineers to assess the reliability of structures and to predict its remaining service life. On the other hand, overdetermined boundary data are crucial to such a geometrical inverse problem. To the best of our knowledge, all cavities identification problems in linear elasticity, investigated in the literature, are based on the essential assumption that overspecified boundary conditions are complete, i.e. both the displacement field and the normal stress are available for the reconstruction of flaws $[5,9,10]$, expect of recent works $[6,22]$ where overdetermined boundary data are incomplete.

Let us mention that the data completion problem mentioned above has been widely investigated (see [11] and references therein). Such a problem can be formulated as follows: given the tractions and the displacement fields on the accessible part of the boundary of the domain of interest, one aims to evaluate the same information on the inaccessible part of the boundary. Needless to say, this ill-posed problem has already been strongly studied with a lot of methods which can be grouped as methods based on the minimization of an energy-like functional [1,2] via the Steklov Poincaré operator, quasi reversibility method [13], iterative methods [18,26,27], iterative regularization methods (namely, relaxation procedures for alternating iterative algorithms) [28,29] and Tikhonov regularization [16]. We refer the reader to [11]. 
In some situations, no information about shear stress (namely the tangential component of the normal stress) is available and only the displacement field and the normal component of the normal stress are accessible. We refer, here, to a sub-Cauchy problem, that is partially overdetermined boundary data [6,22], which consists the main motivation of this paper. Such non-standard situation derives for example from the devices used to compute measurements on the exterior boundary of the mechanical components.

Motivated by the recent results of [8] obtained to recover lacking boundary data via partially overdetermined boundary data and results of [5] obtained to identify cavities from overdetermined boundary data; both results obtained in linear elasticity, we are concerned in this work with a geometrical inverse problem related to the identification of cavities in mechanical structures from partially overdetermined boundary data.

Problem statement: Let $\mathscr{D}$ be a bounded, connected and open set of $\mathbb{R}^{2}$ with a Lipschitz boundary $\Gamma$ and $\omega$ be a subdomain of $\mathscr{D}$ such that $\bar{\omega} \subset \mathscr{D}$. We consider the domain $\Omega=\mathscr{D} \backslash \bar{\omega}$ as a reference configuration of a homogeneous and isotropic elastic material and the subdomain $\omega$ as a void to be recovered. The present topological situation is depicted in Fig. 1. The displacement field $\mathbf{u}$ satisfies the following direct problem

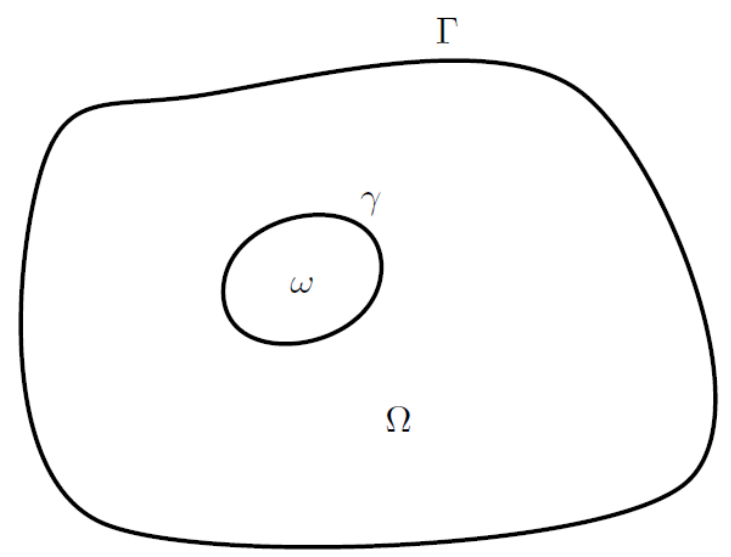

Fig. 1: The computational domain.

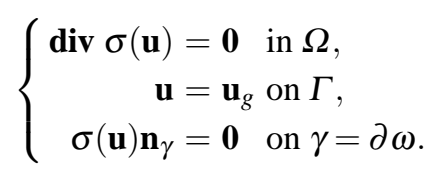

Here, $\sigma(\mathbf{u})$ is the Cauchy stress tensor associated with the displacement field $\mathbf{u}$ and $\varepsilon(\mathbf{u})$ is the linearized strain tensor given by

$$
\varepsilon(\mathbf{u})=\frac{1}{2}\left(\nabla \mathbf{u}+\nabla \mathbf{u}^{t}\right) .
$$

$\sigma$ and $\varepsilon$ are related by the Hooke constitutive law via

$$
\sigma(\mathbf{u})=\lambda(\operatorname{tr} \varepsilon(\mathbf{u})) \mathbf{I}+2 \mu \varepsilon(\mathbf{u})
$$

and conversely

$$
\varepsilon(\mathbf{u})=\frac{1+v}{E} \sigma(\mathbf{u})-\frac{v}{E}(\operatorname{tr} \sigma(\mathbf{u})) \mathbf{I}
$$


Above, tr denotes the trace of matrix, I denotes the identity matrix and $\lambda, \mu$ are the Lamé coefficients related to Young's modulus $E$ and Poisson's ratio $v$ via

$$
\mu=\frac{E}{2(1+v)} \quad \text { and } \quad \lambda=\frac{E v}{(1-2 v)(1+v)} .
$$

$\mathbf{n}_{\gamma}$ denotes the outward unit normal to the boundary of $\omega$ on $\gamma$.

The geometric inverse problem under consideration can be stated as follows : Given the displacement component $\mathbf{u}_{g}$ on $\Gamma$ and measuring the normal traction $\mathbf{T}_{m} \cdot \mathbf{n}$ on $\Gamma$, identify the shape $\gamma$ of $\omega$.

Classical Cauchy problems have been extensively studied by many authors in order to substantiate the uniqueness of the inverse problems. Almost all the proofs rely on Holmgren's type uniqueness theorem. For the first time, it was introduced by Isakov [21] to prove the uniqueness of a discontinuous conductivity coefficient. Then, this method has been used for various classes of inverse problems. In our context, we refer to the work of Ang and al. [3] who proved a uniqueness result for the location and the shape of a cavity with an edge under a non-vanishing traction hypothesis. The same question has been investigated by Ben Ameur [10]. In this work, the main contribution is the treatment of partially overspecified boundary data when one seeks an identifiability issue for voids.

The present research aims to develop an iterative method based on coupling the Steklov Poincaré operator and the shape gradient approach combined with the level set method through the minimization of an energy-like functional [4,5,6,8, $23,22]$ to numerically solve the inverse problem. However, it should be noted that some results related to the detection of cracks [7,15] and obstacles [13] for the Laplace problem are achieved through iterative methods in the case of a Cauchy problem. Indeed, a two-step recovery algorithm, based on least squares fit with an iterated Tikhonov regularization to extend the available data to the whole external boundary as a first step and on the reciprocity gap to retrieve the crack as a second step, was proposed in [15]. While in [7], the recovery procedure was based firstly on building an extension of the available data to the whole boundary using constructive approximation techniques in classes of analytic and meromorphic functions and secondly on the reciprocity gap technique as it was the case in [15]. The approach proposed in [13] was based on an iterative procedure which consists in coupling the method of quasi-reversibility [24] and a simple level set method.

The overview of the paper is as follows. In the upcoming section, we discuss the identifiability issue for the voids identification problem from an incomplete boundary measurements. In the third section, we introduce the shape gradient to identify the cavities from complete data. In the fourth section, we investigate the inverse problem of recovering the shear stress from the knowledge of partially overdetermined boundary data on the external boundary of the domain of interest. The first issue in the fifth section is a brief presentation of the level set method whilst the second one is devoted to the description of the algorithm to be implemented. The sixth section concerns the numerical results. Finally, some concluding remarks and possible future work are reported.

\section{Voids Identifiability}

In the following, we intend to deal with the more general problem of linear elasticity theory in which the specified overdetermined boundary data are the values of the displacement and the traction over an arbitrarily portion of the boundary, using the Muskhelishvili theorem for the plane elasticity and the Almansi lemma for the three-dimensional elasticity to prove the uniqueness question. In this work, we seek for conditions that guarantee that the difference of two possible displacement fields satisfying the same partially overspecified boundary data is either the null solution or at most a rigid body displacement. 
In the recent literature, a non-standard type of problem has attracted considerable interest because it serves physically an auxiliary role. Up to our knowledge, the first one who treated such kind of problems is Hedenmalm. Recently, he has proven a unicity theorem for the bilaplacien operator from partially overdetermined boundary data [20]. For the sub-Cauchy elasticity problems, the question is still open.

However, on an isotropic half-plane, some insights into the question of uniqueness can be gained using a simple calculus, namely an integral representation [14], such that the sub-Cauchy problem reduces to the Cauchy problem for which the uniqueness condition, that is, the unique (null) solution of the homogeneous Cauchy problem, is ensured by Muskhelishvili's theorem. Remarkably, theorems for the half-plane impose much weaker restrictions on the elasticities than the corresponding theorems for domains of arbitrarily shape.

The main result of this section asserts that there is at most one void in the elastic solid which yields the same surface measurements on an arbitrarily small plane portion of the outer boundary. The key to the proof is the unique continuation of the isotropic Lamé system [17] and the Muskhelishvili theorem [31].

Theorem 1. Let $\omega^{1}$ and $\omega^{2}$ be two voids such that $\omega^{1} \cap \omega^{2} \neq \emptyset$ with $C^{1}$-smooth boundary $\gamma^{1,2}=\partial \omega^{1,2}$. For $i=1,2$, let $\mathbf{u}^{i}$ be the solution of the problem (1) defined in $\Omega^{i}=\mathscr{D} \backslash \overline{\omega^{i}}$ such that $\mathbf{u}^{1} \in\left[H^{2}\left(\Omega^{1}\right)\right]^{2}$. Then, if $\omega^{1}$ and $\omega^{2}$ both lead to the same measured normal traction on an open plane portion $M$ of the outer boundary $\Gamma$, namely $\mathbf{n} \cdot \sigma\left(\mathbf{u}^{1}\right) \mathbf{n}=\mathbf{n} \cdot \sigma\left(\mathbf{u}^{2}\right) \mathbf{n}=$ $\mathbf{T}_{m} \cdot \mathbf{n}$ on $M$, we have $\omega^{1}=\omega^{2}$.

Proof. Let $\Omega^{e} \subset \mathscr{D}$ be the external connected component of $\Omega^{1} \cap \Omega^{2}$ such that $\partial \Omega^{e} \subset \gamma^{1} \cup \gamma^{2} \cup \Gamma$ (the one having M as part of its boundary). Let $\mathbf{w}:=\mathbf{u}^{1}-\mathbf{u}^{2}$ be the solution of the following problem

$$
\left\{\begin{aligned}
\operatorname{div} \sigma(\mathbf{w}) & =0 \text { in } \Omega^{e} \\
\mathbf{n} \cdot \sigma(\mathbf{w}) \mathbf{n} & =0 \text { on } \mathrm{M} \\
\mathbf{w} & =\mathbf{0} \text { on } \mathrm{M}
\end{aligned}\right.
$$

Since $\Omega^{e}$ is connected, $\mathbf{w}$ vanishes in the whole domain $\Omega^{e}$ by the Muskhelishvili theorem. Then

$$
\mathbf{u}^{1}=\mathbf{u}^{2} \text { in } \Omega^{e}
$$

Now, we prove by contradiction that $\omega^{1}=\omega^{2}$. Let us so suppose that $\omega^{1} \neq \omega^{2}$. Since numerous possibilities exist for the void, see Fig. 2 for one particular situation, we assume that $\mathscr{O}$ is one of the connected components of $\Omega^{1} \backslash \Omega^{2}$. Subsequently, we have that $\partial \mathscr{O}$ is a union of a finite number of open $C^{1}$-smooth curves. So, for all $x \in \partial \mathscr{O}$, there are only two cases to happen

(1) $x \in \gamma^{1}$.

(2) $x \in \partial \Omega^{e} \cap\left(\gamma^{2} \backslash\left(\gamma^{1} \cup \Gamma\right)\right)$.

In the first case, we have

$$
\sigma\left(\mathbf{u}^{1}\right) \mathbf{n}_{\gamma^{1}}=\mathbf{0} \text { on } \gamma^{1}
$$

where $\mathbf{n}_{\gamma^{1}}$ denotes the outward unit normal to the boundary $\gamma^{1}$.

In the second case, we have by the regularity assumptions

$$
\mathbf{u}^{1}(x)=\mathbf{u}^{2}(x) \quad \text { and so } \quad \sigma\left(\mathbf{u}^{1}(x)\right)=\sigma\left(\mathbf{u}^{2}(x)\right) .
$$




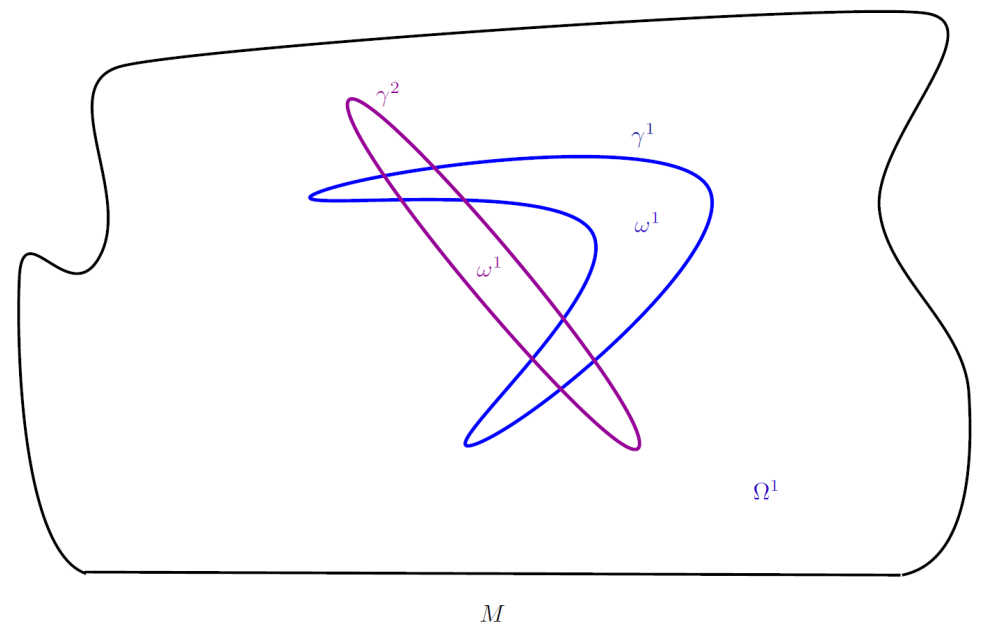

Fig. 2: The connected components of $\Omega^{1} \backslash \Omega^{2}$.

Since $x \in \partial \Omega^{e} \cap\left(\gamma^{2} \backslash\left(\gamma^{1} \cup \Gamma\right) \subset \gamma^{2}\right)$, we have

$$
\sigma\left(\mathbf{u}^{2}\right) \mathbf{n}_{\gamma^{2}}=\mathbf{0} \text { on } \gamma^{2}
$$

where $\mathbf{n}_{\gamma^{2}}$ denotes the outward unit normal to the boundary $\gamma^{2}$.

By (2) and (3), we get that $\mathbf{u}^{1}$ is the solution of the following problem in the open set $\mathscr{O}$

$$
\left\{\begin{array}{l}
\operatorname{div} \sigma\left(\mathbf{u}^{1}\right)=\mathbf{0} \text { in } \mathscr{O} \\
\sigma\left(\mathbf{u}^{1}\right) \mathbf{n}_{\gamma}=\mathbf{0} \text { on } \partial \mathscr{O} .
\end{array}\right.
$$

From the Green formula, we get

$$
\int_{\mathscr{O}} \sigma\left(\mathbf{u}^{1}\right): \varepsilon\left(\mathbf{u}^{1}\right) \mathrm{d} x=\int_{\partial \mathscr{O}} \sigma\left(\mathbf{u}^{1}\right) \mathbf{n}_{\gamma} \cdot \mathbf{u}^{1} \mathrm{~d} s=0 .
$$

We conclude that $\mathbf{u}^{1}$ is a rigid displacement in $\mathscr{O}$. By the unique continuation theorem, we deduce that $\mathbf{u}^{1}$ is a rigid displacement in the whole domain $\Omega^{1}$ which is in contradiction with the hypothesis of the load which is not identically equal to zero $\left(\mathbf{T}_{m} \cdot \mathbf{n} \neq 0\right)$. Hence, $\omega^{1}=\omega^{2}$.

Remark. For the case of disjoint voids, the proof has the same spirit as the one presented above and for the case of monotonous cavities the unicity was proved using the energy functional [22].

\section{Voids Identification}

In this part, we only outline briefly the cavities identification method from overdetermined boundary data. We refer to [5] for a comprehensive analysis. In this section, we aim to recover the void $\omega$, that is to solve the geometrical inverse problem provided that the shear stress, namely $\mathbf{T}_{m} \cdot \tau$ is known on $\Gamma$, where $\tau$ is the unit tangent vector to $\Gamma$. The reconstruction problem of the shear stress $\mathbf{T}_{m} \cdot \tau$ will be addressed in the next section. In order to solve this problem, we follow the same method investigated in [5]. Indeed, we propose a Dirichlet-Neumann approach by the means of a self-regularization technique namely the Kohn-Vogelius formulation [5]. More precisely, we introduce two well-posed problems, with a couple of solutions $\left(\sigma_{\mathrm{D}}, \mathbf{u}_{\mathrm{D}}\right)$ and $\left(\sigma_{\mathrm{N}}, \mathbf{u}_{\mathrm{N}}\right)$ defined in $\Omega$, each of them satisfying the elasticity equations in $\Omega$ as well as 
a homogeneous Neumann boundary condition on $\gamma$. We assign to the first problem a Dirichlet condition (4) and to the second one a Neumann condition (5) both of them on the boundary $\Gamma$ as follows

$$
\begin{aligned}
& \left\{\begin{array}{rlrl}
\operatorname{div} \sigma_{\mathrm{D}} & =\mathbf{0} & & \text { in } \Omega, \\
\varepsilon_{\mathrm{D}} & =\frac{1+v}{E} \sigma_{\mathrm{D}}-\frac{v}{E}\left(\operatorname{tr} \sigma_{\mathrm{D}}\right) \mathbf{I} & \text { in } \Omega, \\
\mathbf{u}_{\mathrm{D}} & =\mathbf{u}_{g} & & \text { on } \Gamma, \\
\sigma_{\mathrm{D}} \mathbf{n}_{\gamma} & =\mathbf{0} & & \text { on } \gamma,
\end{array}\right. \\
& \left\{\begin{array}{rlrl}
\operatorname{div} \sigma_{\mathrm{N}} & =\mathbf{0} & & \text { in } \Omega, \\
\varepsilon_{\mathrm{N}} & =\frac{1+v}{E} \sigma_{\mathrm{N}}-\frac{v}{E}\left(\operatorname{tr} \sigma_{\mathrm{N}}\right) \mathbf{I} & \text { in } \Omega, \\
\sigma_{\mathrm{N}} \mathbf{n} & =\mathbf{T}_{m} & & \text { on } \Gamma, \\
\sigma_{\mathrm{N}} \mathbf{n}_{\gamma} & =\mathbf{0} & & \text { on } \gamma .
\end{array}\right.
\end{aligned}
$$

Let us define the following spaces

$$
L_{s}^{2}(\Omega)=\left\{\alpha=\left(\alpha_{i j}\right) \in\left[L^{2}(\Omega)\right]^{4} ; \alpha_{i j}=\alpha_{j i}\right\}
$$

and

$$
V_{\mathrm{D}}=\left\{\mathbf{v} \in\left[H^{1}(\Omega)\right]^{2} ; \mathbf{v}=\mathbf{0} \text { on } \Gamma\right\}
$$

the bilinear symmetric form $a: L_{s}^{2}(\Omega) \times L_{s}^{2}(\Omega) \rightarrow \mathbb{R}$ and the bilinear form $b: L_{s}^{2}(\Omega) \times\left[H^{1}(\Omega)\right]^{2} \rightarrow \mathbb{R}$ by

$$
a(\sigma, \alpha)=\int_{\Omega}\left[\frac{1+v}{E} \operatorname{tr}(\sigma \alpha)-\frac{v}{E} \operatorname{tr}(\sigma) \operatorname{tr}(\alpha)\right] \mathrm{d} x \quad \text { and } \quad b(\alpha, \mathbf{v})=-\int_{\Omega} \operatorname{tr}(\alpha \nabla \mathbf{v}) \mathrm{d} x
$$

The variational formulations of the Dirichlet problem (4), respectively the Neumann problem (5) are

$$
\left\{\begin{array}{l}
\text { Find }\left(\sigma_{\mathrm{D}}, \mathbf{u}_{\mathrm{D}}\right) \in L_{s}^{2}(\Omega) \times\left[H^{1}(\Omega)\right]^{2} ; \mathbf{u}_{\mathrm{D}}=\mathbf{u}_{g} \text { on } \Gamma \text { such that } \\
a\left(\sigma_{\mathrm{D}}, \alpha\right)+b\left(\alpha, \mathbf{u}_{\mathrm{D}}\right)=0, \quad \forall \alpha \in L_{s}^{2}(\Omega) \\
b\left(\sigma_{\mathrm{D}}, \mathbf{v}\right)=0, \quad \forall \mathbf{v} \in V_{\mathrm{D}}
\end{array}\right.
$$

respectively

$$
\left\{\begin{array}{l}
\text { Find }\left(\sigma_{\mathrm{N}}, \mathbf{u}_{\mathrm{N}}\right) \in L_{s}^{2}(\Omega) \times\left[H^{1}(\Omega)\right]^{2} \text { such that } \\
a\left(\sigma_{\mathrm{N}}, \alpha\right)+b\left(\alpha, \mathbf{u}_{\mathrm{N}}\right)=0, \quad \forall \alpha \in L_{s}^{2}(\Omega) \\
b\left(\sigma_{\mathrm{N}}, \mathbf{v}\right)=-\int_{\Gamma} \mathbf{T}_{m} \cdot \mathbf{v}, \quad \forall \mathbf{v} \in\left[H^{1}(\Omega)\right]^{2}
\end{array}\right.
$$

We adopt above the formulation in two fields, namely the Hellinger-Reissner principle [19]. It consists to write separately the equilibrium equation and the Hooke law in variational form [5].

\subsection{Asymptotic expansions}

Let $\mathscr{U}$ be an open and bounded domain containing $\bar{\Omega}$ and let $\mathbf{F}_{\mathrm{t}}$ be a perturbation of the identity operator, defined by $\mathbf{F}_{\mathrm{t}}=\mathbf{i d}+\mathrm{th}$, where $\mathbf{h}$ is a deformation field belonging to the following space

$$
\mathscr{S}=\left\{\mathbf{h} \in C^{1,1}\left(\overline{\mathscr{U}}, \mathbb{R}^{2}\right) ; \mathbf{h}=\mathbf{0} \text { on } \partial \mathscr{U} \cup \Gamma\right\} .
$$


The condition $\mathbf{h}_{\mid \Gamma}=\mathbf{0}$ means that the boundary $\Gamma$ is clamped during the iterative process $\left(\Gamma=\mathbf{F}_{\mathrm{t}}(\Gamma)\right)$. For sufficiently small t, $\mathbf{F}_{\mathrm{t}}$ is a $C^{1,1}$ diffeomorphism from $\Omega$ onto its image. Let us define the family of domains $\left\{\Omega_{t}\right\}$ and $\left\{\gamma_{t}\right\}$ by

$$
\Omega_{\mathrm{t}}=\mathbf{F}_{\mathrm{t}}(\Omega) \quad \text { and } \quad \gamma_{\mathrm{t}}=\mathbf{F}_{\mathrm{t}}(\gamma)
$$

as well as the following forms

$$
\dot{a}(\sigma, \alpha)=\int_{\Omega} \operatorname{div} \mathbf{h}\left[\frac{1+v}{E} \operatorname{tr}(\sigma \alpha)-\frac{v}{E} \operatorname{tr}(\sigma) \operatorname{tr}(\alpha)\right] \mathrm{d} x
$$

and

$$
\dot{b}(\alpha, \mathbf{v})=-\int_{\Omega}(\operatorname{div} \mathbf{h}) \operatorname{tr}(\alpha \nabla \mathbf{v}) \mathrm{d} x+\int_{\Omega} \operatorname{tr}\left(\alpha\left(\nabla \mathbf{h}^{t} \nabla \mathbf{v}\right)\right) \mathrm{d} x .
$$

Let us consider in $L_{s}^{2}\left(\Omega_{t}\right) \times\left[H^{1}\left(\Omega_{t}\right)\right]^{2},\left(\sigma_{\mathrm{Dt}}, \mathbf{u}_{\mathrm{Dt}}\right)$, respectively $\left(\sigma_{\mathrm{Nt}}, \mathbf{u}_{\mathrm{Nt}}\right)$ the solution of the problem (4), respectively (5) defined on the perturbed domain $\Omega_{t}$ where

$$
L_{s}^{2}\left(\Omega_{t}\right)=\left\{\alpha=\left(\alpha_{i j}\right) \in\left[L^{2}\left(\Omega_{t}\right)\right]^{4} ; \alpha_{i j}=\alpha_{j i}\right\}
$$

The following theorems establish the differentiability of $\mathrm{t} \longmapsto\left(\sigma_{\mathrm{D}}^{\mathrm{t}}, \mathbf{u}_{\mathrm{D}}^{\mathrm{t}}\right)$ and $\mathrm{t} \longmapsto\left(\sigma_{\mathrm{N}}^{\mathrm{t}}, \mathbf{u}_{\mathrm{N}}^{\mathrm{t}}\right)$ where $\left(\sigma_{\mathrm{D}}^{\mathrm{t}}, \mathbf{u}_{\mathrm{D}}^{\mathrm{t}}\right)$ and $\left(\sigma_{\mathrm{N}}^{\mathrm{t}}, \mathbf{u}_{\mathrm{N}}^{\mathrm{t}}\right)$ are the solutions transported to the fixed domain $\Omega$ by

$$
\mathbf{u}_{\mathrm{D}}^{\mathrm{t}}=\mathbf{u}_{\mathrm{Dt}} \circ \mathbf{F}_{\mathrm{t}} \quad \text { and } \quad \sigma_{\mathrm{D}}^{\mathrm{t}}=\sigma_{\mathrm{Dt}} \circ \mathbf{F}_{\mathrm{t}}
$$

and

$$
\mathbf{u}_{\mathrm{N}}^{\mathrm{t}}=\mathbf{u}_{\mathrm{Nt}} \circ \mathbf{F}_{\mathrm{t}} \quad \text { and } \quad \sigma_{\mathrm{N}}^{\mathrm{t}}=\sigma_{\mathrm{Nt}} \circ \mathbf{F}_{\mathrm{t}} .
$$

Theorem 2. [5] The map $t \longmapsto\left(\sigma_{D}^{t}, \mathbf{u}_{D}^{t}\right)$ is continuously differentiable in a neighborhood of 0 and we have that $\left(\dot{\sigma}_{D}, \dot{\mathbf{u}}_{D}\right) \in$ $L_{s}^{2}(\Omega) \times\left[H^{1}(\Omega)\right]^{2}$ is the solution of the following variational problem

$$
\left\{\begin{array}{l}
\text { Find }\left(\dot{\sigma}_{D}, \dot{\mathbf{u}}_{D}\right) \in L_{s}^{2}(\Omega) \times\left[H^{1}(\Omega)\right]^{2} ; \dot{\mathbf{u}}_{D}=\mathbf{0} \text { on } \Gamma \text { such that } \\
a\left(\dot{\sigma}_{D}, \alpha\right)+b\left(\alpha, \dot{\mathbf{u}}_{D}\right)=L_{D}(\alpha), \quad \forall \alpha \in L_{s}^{2}(\Omega) \\
b\left(\dot{\sigma}_{D}, \mathbf{v}\right)=-\dot{b}\left(\sigma_{D}, \mathbf{v}\right), \quad \forall \mathbf{v} \in V_{D}
\end{array}\right.
$$

where

$$
L_{D}(\alpha)=-\dot{a}\left(\sigma_{D}, \alpha\right)-\dot{b}\left(\alpha, \mathbf{u}_{D}\right)
$$

A similar result can be expected for the Neumann problem.

Theorem 3.[5] The map $t \longmapsto\left(\sigma_{N}^{t}, \mathbf{u}_{N}^{t}\right)$ is continuously differentiable in a neighborhood of 0 and we have that $\left(\dot{\sigma}_{N}, \dot{\mathbf{u}}_{N}\right) \in$ $L_{s}^{2}(\Omega) \times\left[H^{1}(\Omega)\right]^{2}$ is the solution to the following variational problem

$$
\left\{\begin{array}{l}
\text { Find }\left(\dot{\sigma}_{N}, \dot{\mathbf{u}}_{N}\right) \in L_{s}^{2}(\Omega) \times\left[H^{1}(\Omega)\right]^{2} \text { such that } \\
a\left(\dot{\sigma}_{N}, \alpha\right)+b\left(\alpha, \dot{\mathbf{u}}_{N}\right)=L_{N}(\alpha), \quad \forall \alpha \in L_{s}^{2}(\Omega) \\
b\left(\dot{\sigma}_{N}, \mathbf{v}\right)=-\dot{b}\left(\sigma_{N}, \mathbf{v}\right), \quad \forall \mathbf{v} \in\left[H^{1}(\Omega)\right]^{2}
\end{array}\right.
$$

where

$$
L_{N}(\alpha)=-\dot{a}\left(\sigma_{N}, \alpha\right)-\dot{b}\left(\alpha, \mathbf{u}_{N}\right)
$$




\subsection{Shape gradient}

A classical way to solve the voids identification problem is to transform it into a shape optimization problem. Following the same procedure described in [5], we consider an energy gap functional $\mathscr{J}$ depending on the domain $\Omega$ given by

$$
\mathscr{J}(\Omega):=\frac{1}{2} \int_{\Omega}\left(\sigma_{\mathrm{D}}-\sigma_{\mathrm{N}}\right):\left(\varepsilon\left(\mathbf{u}_{\mathrm{D}}\right)-\varepsilon\left(\mathbf{u}_{\mathrm{N}}\right)\right) \mathrm{d} x
$$

We remark that $\mathscr{J}(\Omega)=0$ if and only if there is no misfit between both the Dirichlet and Neumann solutions, that is, when $\sigma_{\mathrm{D}}=\sigma_{\mathrm{N}}$ and $\mathbf{u}_{\mathrm{D}}=\mathbf{u}_{\mathrm{N}}$. The inverse problem can so be formulated as a shape optimization one as follows

$$
\left\{\begin{array}{l}
\text { Find } \Omega \text { such that } \\
\mathscr{J}(\Omega)=\min _{\tilde{\Omega} \subset \mathscr{D}} \mathscr{J}(\tilde{\Omega}) .
\end{array}\right.
$$

In the perturbed domain $\Omega_{\mathrm{t}}$, the functional $\mathscr{J}(8)$ is nothing but

$$
\mathscr{J}\left(\Omega_{\mathrm{t}}\right):=\frac{1}{2} \int_{\Omega_{\mathrm{t}}}\left(\sigma_{\mathrm{Dt}}-\sigma_{\mathrm{Nt}}\right):\left(\varepsilon\left(\mathbf{u}_{\mathrm{Dt}}\right)-\varepsilon\left(\mathbf{u}_{\mathrm{Nt}}\right)\right) \mathrm{d} x .
$$

Then, the directional Eulerian shape derivative of $\mathscr{J}$ at $\Omega$ in the direction of $\mathbf{h}$ is defined as

$$
\mathscr{J}^{\prime}(\Omega, \mathbf{h})=\lim _{\mathrm{t} \rightarrow 0} \frac{\mathscr{J}\left(\Omega_{\mathrm{t}}\right)-\mathscr{J}(\Omega)}{\mathrm{t}} .
$$

This derivative $\mathscr{J}$ is called shape derivative if $\mathscr{J}^{\prime}(\Omega, \mathbf{h})$ exists for all $\mathbf{h} \in \mathscr{S}$ and the mapping $\mathbf{h} \mapsto \mathscr{J}^{\prime}(\Omega, \mathbf{h})$ is a continuous and linear functional in $\mathscr{S}$.

Now, we are able to state the main result.

Theorem 4.[5] The mapping $t \longmapsto \mathscr{J}\left(\Omega_{t}\right)$ is $C^{1}$ in a neighborhood of 0 and its derivative at 0 is given by

$$
\mathscr{J}^{\prime}(\Omega, \mathbf{h})=\int_{\gamma} G\left(\mathbf{h} \cdot \mathbf{n}_{\gamma}\right) d s,
$$

with

$$
G=\frac{1}{2}\left[\left(\sigma_{D}: \varepsilon\left(\mathbf{u}_{D}\right)\right)-\left(\sigma_{N}: \varepsilon\left(\mathbf{u}_{N}\right)\right)\right]
$$

\section{Shear stress reconstruction}

This part concerns the recovery of lacking boundary data, namely the shear stress $\mathbf{T}_{m} \cdot \tau=\tau \cdot \sigma(\mathbf{u}) \mathbf{n}$ on the exterior boundary of the domain of interest from the knowledge of partially overdetermined boundary data. The important point to note here is that, to the best of our knowledge, there are no theoretical studies (existence and uniqueness) regarding this problem despite its great importance in applications.

The problem is formulated mathematically as follows: Given the displacement component $\mathbf{u}_{g}$ on $\Gamma$ and measuring the normal traction $\mathbf{T}_{m} \cdot \mathbf{n}$ on $\Gamma$.

$$
\left\{\begin{aligned}
\operatorname{div} \sigma(\mathbf{u}) & =\mathbf{0} & & \text { in } \Omega \\
\mathbf{u} & =\mathbf{u}_{g} & & \text { on } \Gamma \\
\mathbf{n} \cdot \sigma(\mathbf{u}) \mathbf{n} & =\mathbf{T}_{m} \cdot \mathbf{n} & & \text { on } \Gamma .
\end{aligned}\right.
$$

In order to numerically recover the shear stress, we resort to the same approach proposed in [8] that we briefly present herein. This part is concerned with the Steklov-Poincaré operator carried out to solve the sub-Cauchy problem (11) which 
is nothing more than a data completion problem. One way to solve such a problem is to decompose it through an unknown function $\eta$ as follows

$$
\left(\mathscr{P}_{\mathrm{D}}\right)\left\{\begin{aligned}
\operatorname{div} \sigma_{\mathrm{D}}=\mathbf{0} & \text { in } \Omega, \\
\mathbf{u}_{\mathrm{D}}= & \mathbf{u}_{g} \text { on } \Gamma, \\
\mathbf{u}_{\mathrm{D}}=\eta & \text { on } \Gamma_{f}
\end{aligned}\right.
$$

and

$$
\left(\mathscr{P}_{\mathrm{N}}\right)\left\{\begin{array}{rlrl}
\operatorname{div} \sigma_{\mathrm{N}} & =\mathbf{0} & & \text { in } \Omega, \\
\mathbf{n} \cdot \sigma_{\mathrm{N}} \mathbf{n} & =\mathbf{T}_{m} \cdot \mathbf{n} & \text { on } \Gamma, \\
\mathbf{u}_{\mathrm{N}} \cdot \tau & =\mathbf{u}_{g} \cdot \tau & \text { on } \Gamma \\
\mathbf{u}_{\mathrm{N}} & =\eta & & \text { on } \Gamma_{f},
\end{array}\right.
$$

where $\eta$ is the virtual control defined on the fictif boundary $\Gamma_{f}$ as depicted in Fig. 3. The solutions $\mathbf{u}_{\mathrm{D}}$ and $\mathbf{u}_{\mathrm{N}}$ are functions

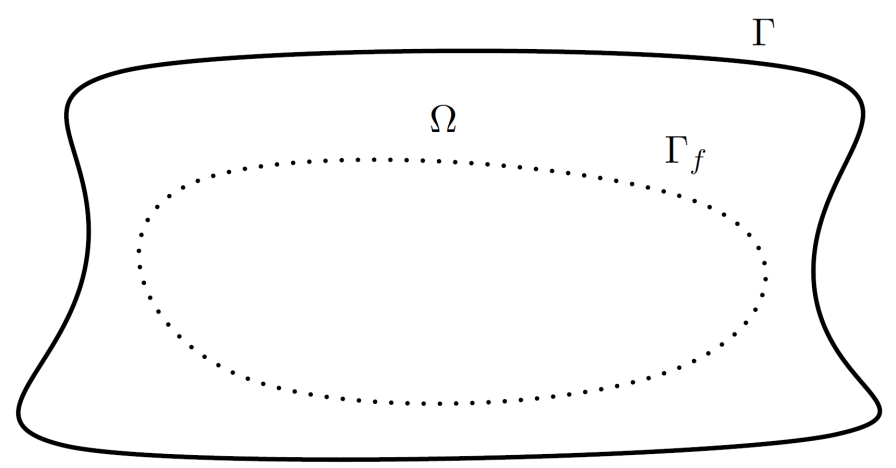

Fig. 3: $\Gamma_{f}$ is a fictif boundary.

of $\eta\left(\mathbf{u}_{\mathrm{D}}=\mathbf{u}_{\mathrm{D}}(\eta)\right.$ and $\left.\mathbf{u}_{\mathrm{N}}=\mathbf{u}_{\mathrm{N}}(\eta)\right)$. The gap between these fields $\mathbf{u}_{\mathrm{D}}$ and $\mathbf{u}_{\mathrm{N}}$ is subsequently minimized with respect to the unknown boundary data $\eta$ in order to produce the desired expanded elastic fields. The gap used herein is the same Kohn-Vogelius error functional investigated in the subsection 3.2 related to voids identification but in this case depending on the virtual control $\eta$, that is

$$
\mathscr{J}(\eta):=\frac{1}{2} \int_{\Omega}\left(\sigma_{\mathrm{D}}-\sigma_{\mathrm{N}}\right):\left(\varepsilon\left(\mathbf{u}_{\mathrm{D}}\right)-\varepsilon\left(\mathbf{u}_{\mathrm{N}}\right)\right) \mathrm{d} x .
$$

The inverse problem is then formulated via the minimization of the energy error functional $\mathscr{J}(14)$ as

$$
\left\{\begin{array}{l}
\text { Find } \eta \text { such that } \\
\mathscr{J}(\eta)=\min _{\tilde{\eta} \in\left[H^{1 / 2}\left(\Gamma_{f}\right)\right]^{2}} \mathscr{J}(\tilde{\eta}) .
\end{array}\right.
$$

The solution $\mathbf{u}_{\mathrm{D}}$ respectively $\mathbf{u}_{\mathrm{N}}$ of the problem (12) respectively (13) can be written as

$$
\mathbf{u}_{\mathrm{D}}=\overline{\mathbf{u}_{\mathrm{D}}}+\mathbf{u}_{\mathrm{D}}^{*} \quad \text { respectively } \quad \mathbf{u}_{\mathrm{N}}=\overline{\mathbf{u}_{\mathrm{N}}}+\mathbf{u}_{\mathrm{N}}^{*},
$$


where $\overline{\mathbf{u}_{\mathrm{D}}}$ and $\overline{\mathbf{u}_{\mathrm{N}}}$ depend on the partially overdetermined boundary data $\left(\mathbf{u}_{g}, \mathbf{T}_{m} \cdot \mathbf{n}\right)$, whilst $\mathbf{u}_{\mathrm{D}}^{*}$ and $\mathbf{u}_{\mathrm{N}}^{*}$ depend on $\eta$ as follows

$$
\left(\mathscr{P}_{\mathrm{D}}^{*}\right)\left\{\begin{array} { r l } 
{ \operatorname { d i v } \sigma _ { \mathrm { D } } ^ { * } = \mathbf { 0 } \text { in } \Omega , } \\
{ \mathbf { u } _ { \mathrm { D } } ^ { * } = \mathbf { 0 } \text { on } \Gamma , } \\
{ \mathbf { u } _ { \mathrm { D } } ^ { * } = \eta \text { on } \Gamma _ { f } , }
\end{array} \quad ( \overline { \mathscr { P } } _ { \mathrm { D } } ) \left\{\begin{array}{rl}
\operatorname{div} \overline{\sigma_{\mathrm{D}}}=\mathbf{0} & \text { in } \Omega, \\
\overline{\mathbf{u}_{\mathrm{D}}} & =\mathbf{u}_{g} \text { on } \Gamma, \\
\overline{\mathbf{u}_{\mathrm{D}}}=\mathbf{0} \text { on } \Gamma_{f}
\end{array}\right.\right.
$$

and

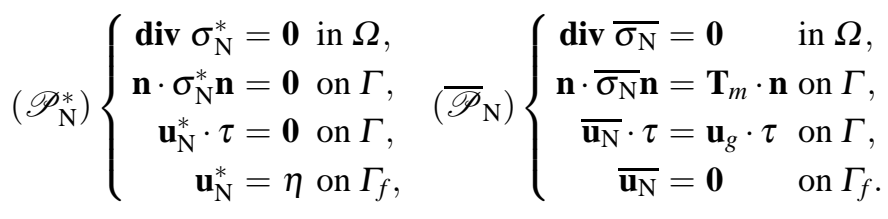

The solution of the problem (15) is recovered if

$$
\sigma_{\mathrm{D}} \mathbf{n}_{f}=\sigma_{\mathrm{N}} \mathbf{n}_{f} \quad \text { on } \Gamma_{f}
$$

According to (16), the condition (17) leads to the boundary equation

$$
\sigma_{\mathrm{D}}^{*} \mathbf{n}_{f}-\sigma_{\mathrm{N}}^{*} \mathbf{n}_{f}=\overline{\sigma_{\mathrm{N}}} \mathbf{n}_{f}-\overline{\sigma_{\mathrm{D}}} \mathbf{n}_{f} \quad \text { on } \Gamma_{f}
$$

Let us introduce some notations useful in the sequel

$$
S_{\mathrm{D}} \eta=\sigma_{\mathrm{D}}^{*} \mathbf{n}_{f}, \quad S_{\mathrm{N}} \eta=\sigma_{\mathrm{N}}^{*} \mathbf{n}_{f} \quad \text { and } \quad \xi=-\left(\overline{\sigma_{\mathrm{D}}} \mathbf{n}_{f}-\overline{\sigma_{\mathrm{N}}} \mathbf{n}_{f}\right) .
$$

Then, one can rewrite the equation (18) as

$$
S \eta=\left(S_{\mathrm{D}}-S_{\mathrm{N}}\right) \eta=\xi \quad \text { on } \Gamma_{f}
$$

where $S$ is the Steklov-Poincaré operator well known in the domain decomposition theory [34].

\section{Numerical analysis}

\subsection{Voids identification}

This part aims to develop an algorithm to numerically solve the shape optimization problem (9). Indeed, the theorem 4 suggests the implementation of a numerical minimization algorithm using the gradient method. We consider for $\mathrm{t} \geq 0$ and $k^{\text {th }}$ iteration, the deformation of $\gamma$, like it was the case in the subsection 3.1

$$
\gamma_{\mathrm{t}}^{k}:=\mathbf{F}_{\mathrm{t}}(\gamma)=\{x+\operatorname{th}(x) ; x \in \gamma\}
$$

where $\mathbf{h}$ is chosen as follows

$$
\mathbf{h} \in \mathscr{S} \quad \text { such that } \quad \mathbf{h}_{\mid \gamma}=-G \mathbf{n}_{\gamma}
$$

$G$ is given by (10). This descent direction (21) guarantees the minimization of the shape functional $\mathscr{J}$ (8). To numerically implement this iterative procedure, we resort to the level set method. It is a numerical technique for tracking shapes, developed in 1988 by Osher and Sethian [33]. During the past few decades, this method has been applied in various fields [32], since it provides a practical way to follow shapest hat change topologies. Let us consider the evolution of a boundary 
$\gamma_{\mathrm{t}}$ (namely a domain $\Omega_{\mathrm{t}} \subset \mathscr{U} \subset \mathbb{R}^{2}$ ) under the velocity field $\mathbf{h}$ (21) related to the sensitivity of the energy error functional $\mathscr{J}(8)$. The basic idea of the level set method is to construct a link between the boundary $\gamma_{\mathrm{t}}(20)$ and a continuous function $\Phi$ defined on the whole domain $\mathscr{U}$. More precisely, the boundary $\gamma_{t}$ can be implicitly represented using the level curve of a function $\Phi$ as follows

$$
\gamma_{\mathrm{t}}=\{x \in \mathscr{U} ; \Phi(x, t)=0\} .
$$

During the process, the cavities to recover will be identified by change in level set function values, with respect to fictitious time t. This change is related to the shape derivative of the functional $\mathscr{J}$. Indeed, the derivation of the equation $\Phi(x, \mathrm{t})=0$ with respect to $t$ leads to the transport equation

$$
\partial_{\mathrm{t}} \Phi+\mathbf{h} \cdot \nabla \Phi=0
$$

Since the normal vector to $\gamma_{\mathrm{t}}$ is given by $\mathbf{n}_{\gamma}=\frac{\nabla \Phi}{|\nabla \Phi|}$ [32], the evolution of $\Phi$ is then governed by the Hamilton-Jacobi equation

$$
\partial_{\mathrm{t}} \Phi+\mathbf{h}_{\mathbf{n}}|\nabla \Phi|=0
$$

where $\mathbf{h}_{\mathbf{n}}$ is the normal velocity. Or, we have chosen $\mathbf{h}=-G \mathbf{n}_{\gamma}=-G \frac{\nabla \Phi}{|\nabla \Phi|}$. As a consequence, the evolution of $\gamma_{\mathrm{t}}$ (namely the evolution of $\Phi$ ) is governed by the so-called level set equation

$$
\left\{\begin{array}{ll}
\partial_{\mathrm{t}} \Phi-G|\nabla \Phi| & =0 \\
\Phi(\cdot, 0) & =\Phi_{0}
\end{array} \quad \text { in } \mathscr{U} \times \mathbb{R}_{+}\right.
$$

where $\Phi_{0}$ is the initial data chosen as the signed distance function to $\gamma_{0}$. Hence, moving the level-set lines along the descent gradient direction $\mathbf{h}$ (21) is equivalent to evolving the function $\Phi$ by solving the level set equation (22).

\subsection{Shear stress reconstruction}

Going back to the resolution of the linear system of equations (19), one can use an iterative preconditioned gradient algorithm which appears to be very efficient. Indeed, at $\mathrm{k}$ iteration, one can reinitialize $\eta$ as

$$
\eta^{k}=\eta^{k-1}+\rho M\left(S \eta^{k-1}-\xi\right)
$$

and the key point, here, relies on choosing the preconditioner $M=S_{D}^{-1}$. Above, $\rho$ is a relaxation coefficient. Hence, each iteration of the algorithm, to be implemented in order to reconstruct the shear stress on $\Gamma$, involves the solution of both problems (12) and (13), to get $S \eta$ and the solution of the following problem

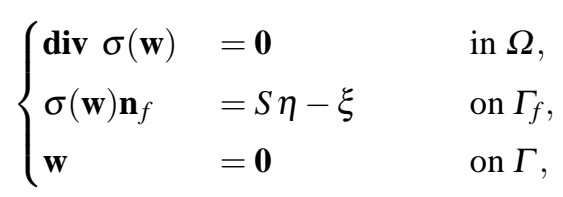

needed to solve the system $S_{D} \chi=S \eta-\xi\left(\chi=\mathbf{w}\right.$ on $\left.\Gamma_{f}\right)$.

\subsection{Algorithm}

Herein, we outline the algorithm to be implemented in order to solve the inverse problem, that is to retrieve the void $\omega$ from the partially overdetermined boundary data $\left(\mathbf{u}_{g}, \mathbf{T}_{m} \cdot \mathbf{n}\right)$ available on $\Gamma$. Let $\Omega^{\text {true }}$ be a domain containing a cavity (or multiple cavities) whose location and shape are to be retrieved from boundary measurements. The partially 
overdetermined boundary data, namely the displacement $\mathbf{u}_{g}$ and the normal component of the normal stress $\mathbf{T}_{m} \cdot \mathbf{n}$ are issued from a numerical computation of a direct problem over the domain $\Omega^{\text {true }}$ containing the cavity(ies) to recover (synthetic data) as follows.

Given $\overline{\mathbf{T}}_{g} \in\left[H^{-\frac{1}{2}}(\Gamma)\right]^{2}$, we solve the direct problem

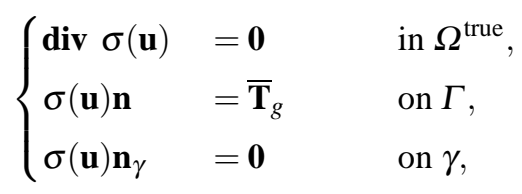

up to a constant. Then, we take $\left(\mathbf{u}_{g}, \mathbf{T}_{m} \cdot \mathbf{n}\right)=\left(\mathbf{u}_{\left.\right|_{\Gamma}},\left(\overline{\mathbf{T}}_{g} \cdot \mathbf{n}\right)_{\left.\right|_{\Gamma}}\right)$. Since the partially overdetermined boundary data $\left(\mathbf{u}_{g}, \mathbf{T}_{m} \cdot \mathbf{n}\right)$ are overspecified, the numerical procedure could be represented in the following algorithm stages. Let us assume that we know the interface $\gamma^{k}$ and a level set function $\Phi^{k}$ associated to $\gamma^{k}$.

\section{(1) Shear stress reconstruction}

(a) Choose an initial data $\eta^{0}$.

(b) Solve the problems (12) and (13) in the domain $\Omega_{k}$, where $\Omega_{k}=\mathscr{D} \backslash \overline{\omega_{k}}$.

(c) Solve the problem (23) and get $\mathbf{w}$.

(d) Let $\eta^{k}=\eta^{k-1}+\rho \mathbf{w}$.

(e) Go back to the first step (a) until the stopping condition:

$\left\|\mathbf{u}_{\mathrm{D}}-\mathbf{u}_{\mathrm{N}}\right\|_{\left[L^{2}(\Omega)\right]^{2}} \leq \varepsilon$ is reached ( $\varepsilon$ is a given tolerance level; in the following section, we will choose $\varepsilon=10^{-2}$ ).

(2) Voids identification

(a) Compute the solutions $\left(\sigma_{\mathrm{D}}, \mathbf{u}_{\mathrm{D}}\right)$ and $\left(\sigma_{\mathrm{N}}, \mathbf{u}_{\mathrm{N}}\right)$ of the problems (4) and (5) in the domain $\Omega_{k}$.

(b) Compute the velocity function $G$ on $\gamma^{k}$, given by (10).

(c) Update the level set function $\Phi^{k}$ by solving the level set equation (22) and get a new function $\Phi^{k+1}$.

(d) Go back to the first step ( 1 a) until the stopping criterion is fulfilled.

It should be noted that the stopping criterion of the algorithm exposed above is

$$
d_{H}\left(\gamma^{k}, \gamma^{k-1}\right)<C(\delta x)^{2}
$$

where $C$ is a constant independent of the grid parameter $\delta x . d_{H}$ denotes the Hausdorff distance defined for two sets $A, B \subset \mathbb{R}^{2}$ by

$$
d_{H}(A, B)=\max \left(\sup _{a \in A} d(a, B), \sup _{b \in B} d(A, b)\right)
$$

where

$$
d(a, B)=\inf _{b \in B}|a-b|
$$

\section{Results}

The purpose of this section is to present numerical results. The domain $\mathscr{U}$ is the square $[-1,1] \times[-1,1]$ and we consider the inverse problem of the identification of two cavities. Indeed, we consider disconnected cavities: the union of the two disjointed circles of radius 0.15 centred at $(-0.35,0)$ and $(0.35,0)$ and a connected initial guess: the circle of radius 0.65 centred at the origin. The initial guess is sufficiently big to include the unknown cavities. The results reported in Fig. 4 are in good agreement with the exact ones and show the flexibility of the proposed methodology to recover multiple cavities even from partially overdetermined boundary data. 

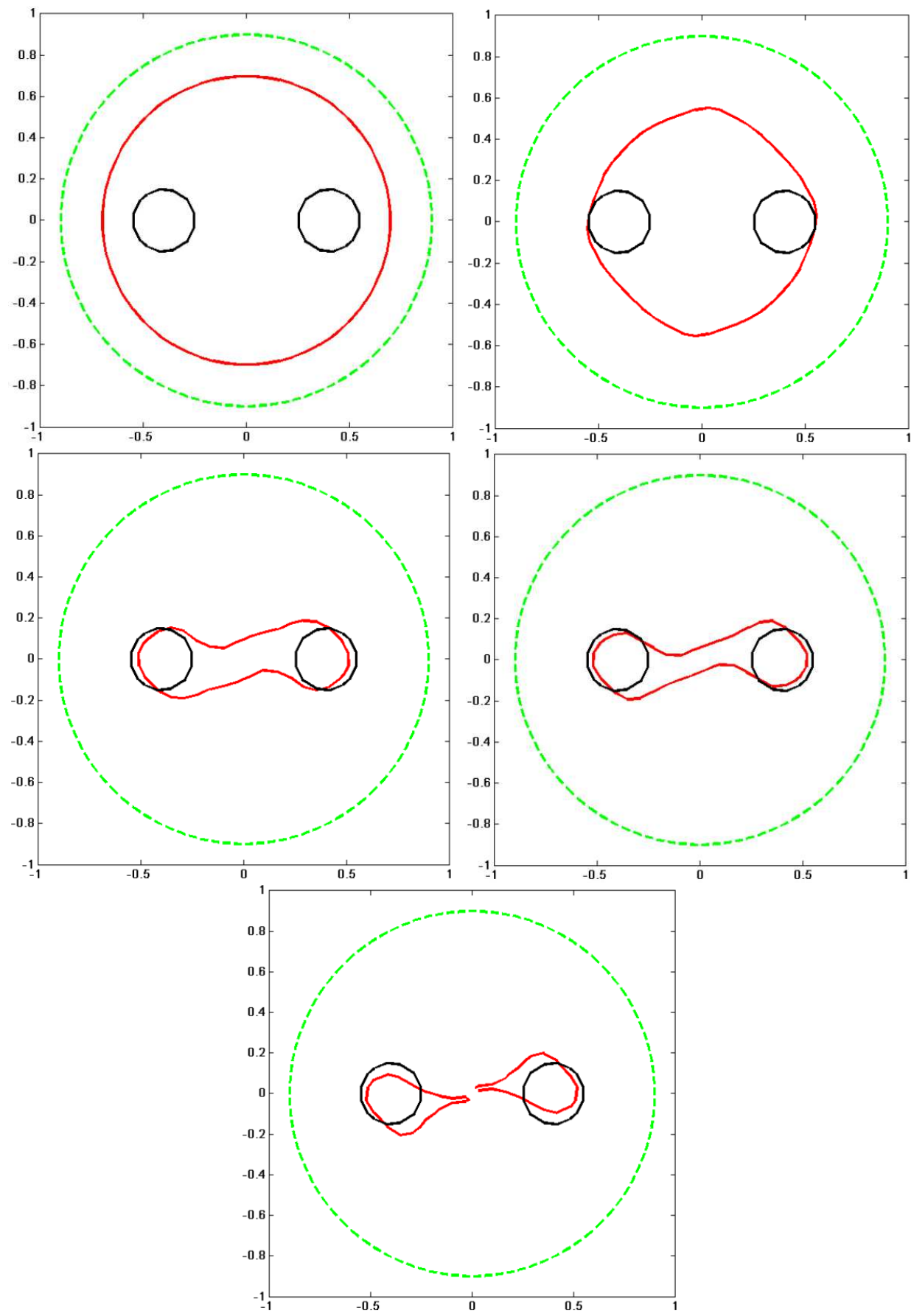

Fig. 4: $\Gamma$ the exterior boundary (the dashed green line), $\gamma$ the exact solution (the black line), the evolution of the boundary $\gamma^{k}$ (the red line) for $k=1,300,434,448,449$ (left to right, top to bottom).

\section{Conclusion}

This paper introduces an iterative method for solving a geometrical inverse problem in linear elasticity. The problem consists in recovering voids from partially overspecified boundary data. The approach proposed combines the resolution of a data completion problem and a cavities identification one. While transforming these both problems into optimization ones, the same energy gap-cost functional is introduced. Numerical simulations have highlighted the efficiency of the 
method. Some open questions such as the existence and the uniqueness of solution concerning the shear stress reconstruction problem deserve an answer. An extension to non-linear elasticity framework could be an interesting future direction of the investigation.

\section{Competing interests}

The authors declare that they have no competing interests.

\section{Authors' contributions}

All authors have contributed to all parts of the article. All authors read and approved the final manuscript.

\section{References}

[1] Andrieux S, Baranger T N 2008, An energy error-based method for the resolution of the Cauchy problem in 3D linear elasticity, Comput. Methods Appl. Mech. Engng. 197, 902-920.

[2] Andrieux S, Baranger T N, Ben Abda A 2006, Solving Cauchy problems by minimizing an energy-like functional, Inverse Problems, 22, no.1, 115-133.

[3] Ang D D, Trong D D, Yamamoto M 1999, Identification of cavities inside two-dimensional heterogeneous isotropic elastic bodies, Journal of Elasticity, 56, 199-212.

[4] Ben Abda A, Bouchon F, Peichl G H, Sayeh M, Touzani R 2013, A Dirichlet-Neumann cost functional approach for the Bernoulli problem, J. Engng. Math. 81, no.1, 157-176.

[5] Ben Abda A, Jaïem E, Khalfallah S, Zine A 2016, An energy gap functional: Cavity identification in linear elasticity, Journal of Inverse and Ill Posed Problems, 25, no.5, 573-595.

[6] Ben Abda A, Jaïem E, Rjaibi B 2016, Cavities identification from partially overdetermined boundary data in linear elasticity, J. Appl. Computat. Math. 5:295. doi:10.4172/2168-9679.1000295

[7] Ben Abda A, Kallel M, Leblond J, Marmorat J P 2002, Line segment crack recovery from incomplete boundary data, Inverse Problems, 18, 1057-1077.

[8] Ben Abda A, Khalfallah S 2016, Lacking data recovery via partially overdetermined boundary conditions in linear elasticity, J. Appl. Mech. Eng. 5: 202. doi:10.4172/2168-9873.1000202.

[9] Ben Ameur H, Burger M, Hackl B 2007, Cavity identification in linear elasticity and thermoelasticity, Math. Methods Appl. Sci. 30, no.6, 625-647.

[10] Ben Ameur H, Burger M, Hackl B 2004, Level set methods for geometric inverse problems in linear elasticity, Inverse Problems, 20, no.3, 673-696.

[11] Bilotta A, Turco E 2009, A numerical study on the solution of Cauchy problem in elasticity, International Journal of Solids and Structures, 46, 4451-4477.

[12] Bonnet M, Constantinescu A 2005, Inverse problems in elasticity, Inverse Problems, 21, no.2, 1-50.

[13] Bourgeois L, Dardé J 2010, A quasi-reversibility approach to solve the inverse obstacle problem, Inverse Problems and Imaging, 4, no.3, 351-377.

[14] Bui H D 1968, Transformation des données aux limites relatives au demi-plan élastique homogène et isotrope, Int. J. Solids Structures. 4, 1025-1030.

[15] Cimetière A, Delvare F, Jaoua M, Kallel M, Pons F 2002, Recovery of cracks from incomplete boundary data, Inverse Problems in Engng, 10, no.4, 377-392.

[16] Cimetière A, Delvare F, Jaoua M, Pons F 2001, Solution of the Cauchy problem using iterated Tikhonov regularization, Inverse Problems, 17, 553-570.

[17] Dehman B, Robbiano L 1993, La propriété du prolongement unique pour un système elliptique. Le système de Lamé, J. Math. Pures Appl. 72, 475-492. 
[18] Delvare F, Cimetière A, Hanus J L, Bailly P 2010, An iterative method for the Cauchy problem in linear elasticity with fading regularization effect, Comput. Methods Appl. Mech. Engng., 199, 3336-3344.

[19] Destuynder P, Djaoua M 1981, Sur une interprétation mathématique de l'intégrale de Rice en théorie de la rupture fragile, Math. Methods. Appli. Sci. 3, no.1, 70-87.

[20] Hedenmalm H 2015, On the uniqueness theorem of Holmgren. Math. Z. no.281, (2015) 357-378.

[21] Isakov V 1988, On uniqueness of recovery of a discontinuous conductivity coefficient, Communications on pure and applied mathematics. 41 , no.7, 865-877

[22] Jaïem E 2016, Shape derivative of an energy error functional for voids detection from sub-Cauchy data, Electronic Journal of Differential Equations, 2016, no.265, 1-13.

[23] Jaïem E, Khalfallah S 2017, An energy-gap cost functional for cavities identification, Nonlinear Studies, 24, no.4, 745-756.

[24] Lattès R, Lions J-L 1967, Méthode de quasi-réversibilité et applications, Dunod, Paris.

[25] Lemaitre J 1996, A Course on Damage Mechanics, Springer, Berlin.

[26] Marin L 2009, The minimal error method for the Cauchy problem in linear elasticity. Numerical implementation for twodimensional homogeneous isotropic linear elasticity, International Journal of Solids and Structures, 46, 957-974.

[27] Marin L, Delvare F, Cimetière A 2015, Fading regularization MFS algorithm for inverse boundary value problems in twodimensional linear elasticity, International Journal of Solids and Structures, 000, 1-12.

[28] Marin L, Johansson B T 2010, A relaxation method of an alternating iterative algorithm for the Cauchy problem in linear isotropic elasticity, Comput. Methods Appl. Mech. Engng., 199, 3179-3196.

[29] Marin L, Johansson B T 2010, Relaxation procedures for an iterative MFS algorithm for the stable reconstruction of elastic fields from Cauchy data in two-dimensional isotropic linear elasticity, International Journal of Solids and Structures, 47, 3462-3479.

[30] Mishnaevsky L 2007, Computational Mesomechanics of Composites: Numerical Analysis of the Effect of Microstructures of Composites on Their Strength and Damage Resistance, John Wiley \& Sons, New York.

[31] Muskhelishvili N I 1977, Some Basic Problems on the Mathematical Theory of Elasticity, Springer, Berlin.

[32] Osher S, Fedkiw R 2002, Level Set Methods and Dynamic Implicit Surfaces, Appl. Math. Sci. 153, Springer, New York.

[33] Osher S, Sethian J A 1988, Fronts propagating with curvature-dependent speed: Algorithms based on Hamilton-Jacobi formulations, J. Comput. Phys. 79, no.1, 12-49.

[34] Quarteroni A, Valli A 1999, Domain Decomposition Methods for Partial Differential Equations, Oxford Science Publications, Oxford. 